\title{
Photoluminescence of carbon in situ doped GaN grown by halide vapor phase epitaxy
}

\author{
R. Zhang and T. F. Kuech ${ }^{\mathrm{a})}$ \\ Department of Chemical Engineering, University of Wisconsin, Madison, Wisconsin 53706
}

(Received 26 November 1997; accepted for publication 2 February 1998)

\begin{abstract}
Carbon was in situ doped into GaN during halide vapor phase epitaxy and photoluminescence properties of the $\mathrm{C}$-doped $\mathrm{GaN}$ film were investigated. It has been found that incorporation of carbon into GaN produces a significant yellow luminescence around $2.2 \mathrm{eV}$. The peak position of the yellow band blueshifts linearly and the intensity of that band monotonically decreases with measurement temperature, with systematic changes in the linewidth. These results suggest that multiple donor-acceptor recombination channels are involved in the yellow luminescence. (C) 1998 American Institute of Physics. [S0003-6951(98)02013-0]
\end{abstract}

Recent advances in GaN-based materials, ${ }^{1}$ such as lightemitting diodes (LEDs), ${ }^{2}$ have stimulated worldwide interest in developing novel GaN-based optoelectronic and electronic devices. Although a GaN continuous-wave (cw) injection laser diode has successfully been operated at room temperature with a lifetime greater than $1000 \mathrm{~h},{ }^{3}$ some persistent problems in GaN material have to be resolved in order to realize higher performance devices. One such problem is the behavior and impact of carbon impurities in GaN. Carbon is one of the most common contaminants in the metalorganic vapor phase epitaxy (MOVPE) of $\mathrm{GaN}^{4}$ Carbon impurities in $\mathrm{GaN}$ are thought to occupy anion sites and act as acceptors. 5,6 Carbon may also combine with other defects, such as $\mathrm{Ga}$ vacancies, ${ }^{7}$ due to the electronegativity and size differences between the carbon impurity and host atom. Carbon impurities are also believed to be related to yellow luminescence (YL) in GaN. ${ }^{7-10}$ Some of the initial photoluminescence (PL) studies on $\mathrm{C}$-doped GaN were carried out by Pankove and Hutchby. ${ }^{7}$ They reported that $\mathrm{C}$-implanted GaN exhibited a strong yellow luminescence band centered around 2.17 $\mathrm{eV}$ at $78 \mathrm{~K}$. This band was attributed to defects arising from implantation damage, since the YL band could also be seen in samples implanted with other elements. ${ }^{7}$ Ogino and Aoki ${ }^{8}$ found that the YL in both $\mathrm{GaN}$ microcrystal powder and needlelike crystals was greatly enhanced by intentional introduction of carbon into those materials. They concluded that the carbon impurities were crucial for YL. The YL was suggested to involve a radiative transition between a shallow donor, with the ionization energy of $\sim 25 \mathrm{meV}$, and a deep acceptor, situated $860 \mathrm{meV}$ above the top of the valence band. ${ }^{8}$ Polyakov et al. ${ }^{9}$ and Niebuhr et al. ${ }^{10}$ have also attributed the observed YL in GaN grown by MOVPE to carbon impurities. The halide vapor phase epitaxy (HVPE) technique does not use carbon containing source materials but only uses $\mathrm{NH}_{3}, \mathrm{HCl}$, and ultrapure $\mathrm{Ga}$ to synthesize GaN. ${ }^{11,12}$ Carbon would only appear in these materials as an unintentional dopant. The YL band in typical HVPE-grown $\mathrm{GaN}$ is either absent or very weak ${ }^{11,12}$ in agreement with the lack of carbon in the growth system. HVPE-grown GaN is

${ }^{a) E l e c t r o n i c ~ m a i l: ~ k u e c h @ e n g r . w i s c . e d u ~}$ therefore a suitable technique to study the correlation between $\mathrm{YL}$ and $\mathrm{C}$ atoms in $\mathrm{GaN}$ by intentional $\mathrm{C}$ doping during GaN growth. In this letter, we will report on the PL properties of $\mathrm{C}$-doped GaN grown by HVPE. We have found that incorporation of $\mathrm{C}$ into $\mathrm{GaN}$ produces significant $\mathrm{YL}$ in these HVPE-grown GaN layers. PL measurements operated at variable temperature show a clear dependence of peak position, peak width, and intensity of YL on the measurement temperature. All results indicate that there are multiple transition channels involved in YL. Comparison of the PL properties of $\mathrm{C}$-doped $\mathrm{GaN}$ films to those of non-C-doped GaN samples have also shown that a variety of recombination mechanisms lead to the appearance of the PL band in the YL energy regime.

All GaN samples were grown on (0001) sapphire substrates in a horizontal atmospheric pressure HVPE system. Details of the reactor and growth technique were described elsewhere. ${ }^{12}$ The reactive gases were injected into the chamber by separate inlets and mixed just above substrate to prevent premature gas phase reactions. $\mathrm{NH}_{3}$ was used as the nitrogen source and the mole fraction of $\mathrm{NH}_{3}$ was kept approximately constant at $X_{\mathrm{NH}_{3}} \cong 0.11$. $\mathrm{HCl}$ was reacted at a high temperature with the liquid $\mathrm{Ga}$ to form $\mathrm{GaCl}$. The $\mathrm{GaCl}$ was transported to the substrate which was held at a higher temperature. Temperature of the Ga boat was kept at $850^{\circ} \mathrm{C}$ while the substrate temperature was $1030^{\circ} \mathrm{C}$. $\mathrm{N}_{2}$ was used as carrier gas. The input ratio of $\mathrm{NH}_{3}$ to $\mathrm{HCl}$ was 30 . The only intentional dopant specie was carbon, supplied as high purity $10 \%$ propane in hydrogen mixture. The propane/ $\mathrm{H}_{2}$ mixture was added to the growth system through a separate $\mathrm{N}_{2}$ buffer line. ${ }^{12}$ The mole fraction of $\mathrm{C}_{3} \mathrm{H}_{8}$ in the reactor was varied from $1.8 \times 10^{-4}$ to $3.3 \times 10^{-3}$. The growth rate under that condition was in the range of $1-1.7 \mu \mathrm{m} / \mathrm{min}$ and the thickness of samples was between 30 and $50 \mu \mathrm{m}$. The surface morphology was dominated by hexagonal surface features and very regular cracks. The cracks were due to the differential thermal expansion between the GaN film and the sapphire substrate.

The double-crystal x-ray diffraction technique was used to characterize the structural properties of the epitaxial films. The rocking-curve measurements gave a range of full width 


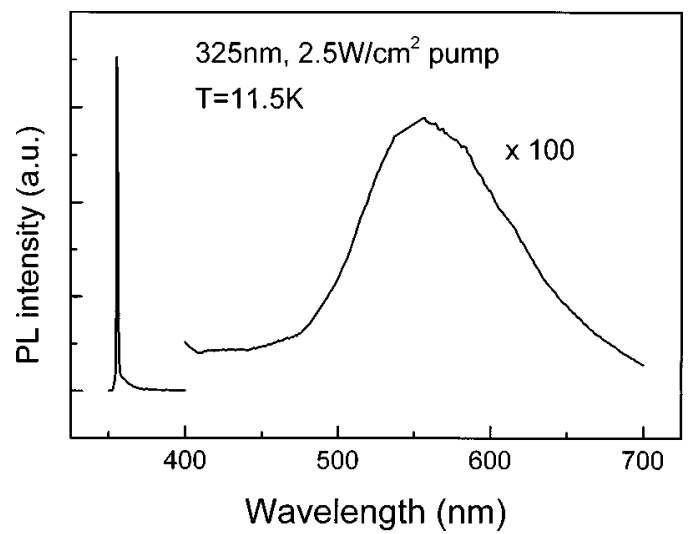

FIG. 1. PL spectrum of intentionally C-doped GaN film on (0001) sapphire substrate grown by HVPE. The measurement was operated at $11.5 \mathrm{~K}$ and under $2.5 \mathrm{~W} / \mathrm{cm}^{2}$ excitation of the $325 \mathrm{~nm}$ line of a HeCd laser. The FWHM of the BE band is $\sim 7.4 \mathrm{meV}$. The $\mathrm{C}$-induced $\mathrm{YL}$ band is identified, but reduced by a factor of $100 \times$ when compared to the $\mathrm{BE}$ band.

half maximum (FWHM) from 495 to 869 arcsec. All samples were $n$ type.

The $\mathrm{C}$ doping efficiency was studied through the use of secondary ion mass spectroscopy (SIMS). Two samples have been tested and compared. One sample was grown by the $\mathrm{NH}_{3}-\mathrm{GaCl}-\mathrm{N}_{2}$ system, while another was grown with the addition of $15 \mathrm{sccm} \mathrm{C}_{3} \mathrm{H}_{8} / \mathrm{H}_{2}$ into the system (named sample A hereafter). The unintentionally doped sample had a measured carbon concentration that was $\leqslant 2 \times 10^{16}$, which is at the detection limit of the SIMS measurement for carbon. The presence of a propane mole fraction of $X_{\mathrm{C}_{3} \mathrm{H}_{8}}=1.8 \times 10^{-4}$ lead to a rapid increase in the carbon content up to a measured value of $5 \times 10^{18} \mathrm{~cm}^{-3}$. The room-temperature Hall measurement on this sample gave an electron concentration of $4 \times 10^{18} \mathrm{~cm}^{-3}$. This result indicates that although carbon is considered to be an acceptor, there is continued compensation of the incorporated carbon by the generation or incorporation of additional shallow donors. These shallow donors continue to dominate the electrical properties of the $\mathrm{GaN}$ film. The measured room-temperature Hall mobility of that sample was $90 \mathrm{~cm}^{2} \mathrm{~V}^{-1} \mathrm{~s}^{-1}$.

Photoluminescence was employed to study the optical properties of GaN samples. The PL was excited by the 325 $\mathrm{nm}$ line of a $\mathrm{HeCd}$ laser and the excitation power intensity was $2.5 \mathrm{~W} / \mathrm{cm}^{2}$. All C-doped samples showed both sharp band edge (BE) luminescence and the YL band at both low and room temperature. A typical low-temperature PL spectrum, where the measurement temperature was $11.5 \mathrm{~K}$, of sample A is presented in Fig. 1. In that figure, the two major luminescence bands are shown with the BE band being most prominent. The FWHM of the BE peak at that temperature is $7.4 \mathrm{meV}$ for this intentionally C-doped $\mathrm{GaN}$ sample. There are several individual bound exciton and free exciton peaks contained within our BE peak. ${ }^{13}$ At very low measurement temperatures, such as $11.5 \mathrm{~K}$ in Fig. 1, the principal contribution to the BE peak should be due to excitons bound to the donors attributed to the $n$-type background doping. At a much lower emission intensity, a broad luminescence band around $560 \mathrm{~nm}$ emerges. The intensity of this YL band is two orders less than that of the BE band under our measurement conditions. This intensity difference is associated with the reduced concentration of defects, relative to MOVPE and Downloaded 04 Jan 2007 to 128.104 .30 .229 . Redistribution subject
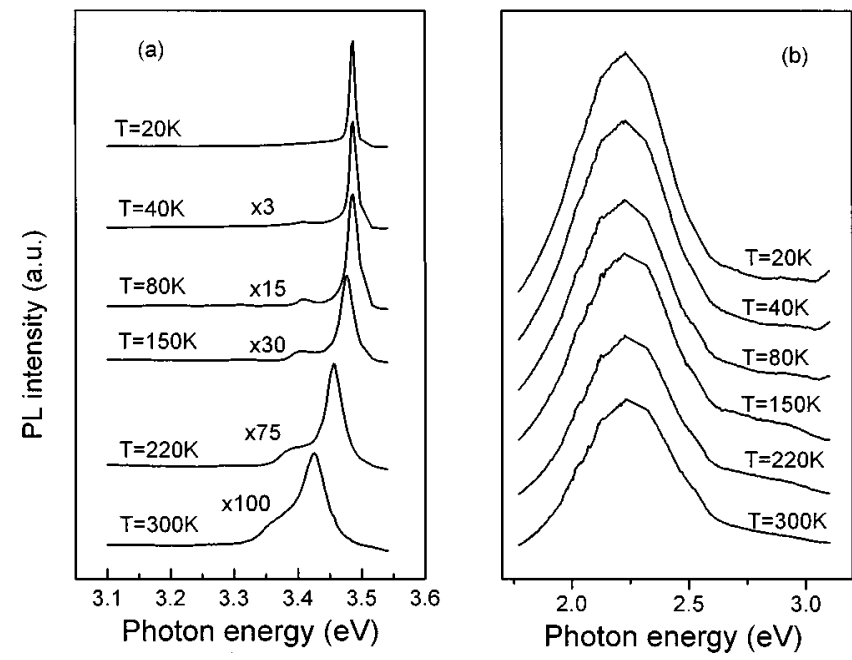

FIG. 2. PL spectra of HVPE-grown C-doped GaN at various measurement temperatures over the energy ranges of 3.1-3.54 eV (a) and 1.75-3.1 eV (b). The peak position, FWHM and intensity of the BE band change dramatically from 20 to $300 \mathrm{~K}$ (a) while those of the YL band change very little over the same temperature range $(\mathrm{b})$.

other techniques grown materials, responsible for the $\mathrm{YL}$ band.

Figures 2(a) and 2(b) display PL spectra of sample A determined over the temperature range of $20-300 \mathrm{~K}$ for the energy ranges of $3.1-3.54 \mathrm{eV}$ and $1.75-3.1 \mathrm{eV}$, respectively. In Fig. 2(a), the BE peak becomes broader and less intense, and the energy position of that peak shifts to low energy side as temperature increases, similar to other results reported in the literature. ${ }^{13}$ At $T=20 \mathrm{~K}$, the BE peak is located at 3.4862 $\mathrm{eV}$ with the FWHM of $9.8 \mathrm{meV}$. At $T=300 \mathrm{~K}$, the BE band moves to $3.4254 \mathrm{eV}$ and the FWHM becomes $53.5 \mathrm{meV}$. The intensity of the $\mathrm{BE}$ luminescence decreases by a factor of about 100 from $20 \mathrm{~K}$ to room temperature. It can also be seen from Fig. 2(a) that another wide emission band centered around 3.35-3.4 eV becomes visible and comparable to the $\mathrm{BE}$ band when the temperature is increased from 20 to 300 K.

The intensity and peak position of the YL bands in Fig. 2(b) are less temperature dependent than the BE band shown in Fig. 2(a). From 20 to $300 \mathrm{~K}$, the intensity of the YL only decreases by half while the peak position shifts $\sim 25 \mathrm{meV}$. The FWHM of the YL band changes very little from about $0.49 \mathrm{eV}$ at $20 \mathrm{~K}$ to $0.475 \mathrm{eV}$ at $300 \mathrm{~K}$. All parameters of the YL band are summarized in Fig. 3. The change in the YL peak position can be well described by a linear law, $\Delta E$ $=1.08 \mathrm{kT}$, which agrees well with the configuration coordinate (CC) model for donor-acceptor pair recombination. ${ }^{14}$ The proportionality factor of 1.08 is, however, almost half of that calculated from data in Ref. 8. The YL band peak position presented here is also substantially different from that reported by Ogino and Aoki, ${ }^{8}$ where the YL peak shifts to a lower energy due to a combining effect of both the temperature dependence of the energy gap and the linear shift expected in the CC model as the measurement temperature increased. These results indicate that the shallow states involved in the YL transition in the HVPE-grown GaN layers are less influenced by the temperature-induced change of the band edge.

The intensity of the YL band in the GaN:C films de-

P license or copyright, see http://apl.aip.org/apl/copyright.jsp 


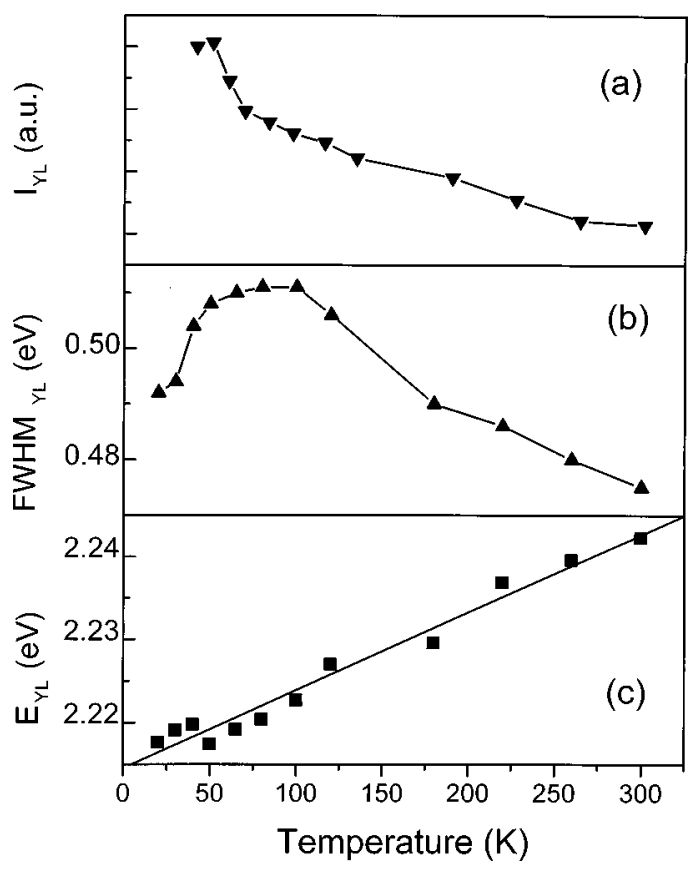

FIG. 3. Temperature dependence of the peak position, FWHM and intensity of the YL band. Note that the peak position shifts in a linear fashion to higher energy, in agreement with a configuration coordinate model of donor-acceptor pair recombination (see Ref. 14). The complex dependence of the YL FWHM on temperature indicates that there are multiple recombination channels involved in the YL band.

creases monotonically from low to high temperature and this dependence cannot be fit a simple single exponential curve. In fitting low-temperature data, an activation energy of 8.6 meV can be obtained. The FWHM of YL band exhibits a complex behavior with temperature, i.e., the FWHM initially increases and then decreases as measurement temperature is increased. The nonexponential behavior of the peak intensity combined with the complex dependence of the FWHM implies that there were multiple recombination channels contributing to the YL band. These channels, arising from different chemical or physical origins, will each have a separate temperature dependence. The peak width and position of the entire YL band will be dependent on the relative concentration and recombination kinetics of these channels leading to a complex behavior with temperature. Our measurements indicate that this defect luminescence band has a complex origin, both chemically and physically.

In order to distinguish whether the $\mathrm{C}_{3} \mathrm{H}_{8}$ or the $\mathrm{H}_{2}$ introduced in the $\mathrm{C}_{3} \mathrm{H}_{8} / \mathrm{H}_{2}$ mixture in the growth system leads to the appearance of the YL band, GaN films were grown by HVPE under the same conditions of the C-doped samples except for the absence of $\mathrm{C}_{3} \mathrm{H}_{8}$ but in the presence of the additional $\mathrm{H}_{2}$ flow and studied by PL. ${ }^{15}$ The typical background carbon concentration in these " $\mathrm{H}_{2}$-doped" samples was determined by SIMS $\sim 4 \times 10^{16} \mathrm{~cm}^{-3}$, which is about two orders lower than the intentional C-doped samples and close to or at the SIMS detection limit. In the " $\mathrm{H}_{2}$-doped" sample, a strong YL band is observable and comparable to the BE band at both low and room temperatures. The variable temperature PL spectra show substantial differences in the YL peak position, intensity, and FWHM when compared to the C-doped samples. These results again support the idea that there are multiple origins for the spectral features in the wavelength regime associated with the YL band. The YL band can be induced by a variety of mechanisms with the features of the YL band that are C-doping related presented here. The detailed characteristics of the " $\mathrm{H}_{2}$-doped" samples will be presented elsewhere.

In conclusion, photoluminescence properties of in situ $\mathrm{C}$-doped GaN grown by HVPE have been studied. We have found that direct and controlled incorporation of carbon into $\mathrm{GaN}$ can produce a significant YL band. The energy position of the YL band blueshifts linearly and the intensity of the YL band decreases monotonically with temperature. The temperature dependence of the FWHM of the YL peak implies that there are multiple channels related to the YL band in these films. Comparison of the PL properties of C-doped $\mathrm{GaN}$ films to those of $\mathrm{GaN}$ samples grown in the presence of additional $\mathrm{H}_{2}$ in the growth ambient have shown that the PL features of the YL range can originate from different mechanisms.

The authors would like to acknowledge financial support of the Naval Research Laboratory, the DARPA URI visible light emitters, and the facilities support of the NSF Materials Research Science and Engineering Center on Nanostructured Materials and Interfaces.

${ }^{1}$ S. Nakamura, M. Senoh, and T. Mukai, Jpn. J. Appl. Phys., Part 2 30, L1708 (1991).

${ }^{2}$ S. Nakamura, T. Mukai, and M. Senoh, J. Appl. Phys. 76, 8189 (1994).

${ }^{3}$ The lifetime of the GaN laser has been continuously improved and updated in conferences. The latest unpublished lifetime is $1800 \mathrm{~h}$.

${ }^{4}$ P. de Mierry, O. Ambacher, H. Kratzer, and M. Stutzmann, Phys. Status Solidi A 158, 587 (1996).

${ }^{5}$ J. C. Zolper, R. G. Wilson, S. J. Pearton, and R. A. Stall, Appl. Phys. Lett. 68, 1945 (1996).

${ }^{6}$ C. R. Abernathy, J. D. MacKenzie, S. J. Pearton, and W. S. Hobson, Appl. Phys. Lett. 66, 1969 (1995).

${ }^{7}$ J. I. Pankove and J. A. Hutchby, J. Appl. Phys. 47, 5387 (1976).

${ }^{8}$ T. Ogino and M. Aoki, Jpn. J. Appl. Phys. 19, 2395 (1980).

${ }^{9}$ A. Y. Polyakov, M. Shin, J. A. Freitas, M. Skowronski, D. W. Greve, and R. G. Wilson, J. Appl. Phys. 80, 6349 (1996).

${ }^{10}$ R. Niebuhr, K. Bachem, K. Bombrowski, M. Maier, W. Pletschen, and U. Kaufmann, J. Electron. Mater. 24, 1531 (1995).

${ }^{11}$ R. J. Molnar, K. B. Nichols, P. Maki, E. R. Brown, and I. Melngailis, Mater. Res. Soc. Symp. Proc. 378, 479 (1995).

${ }^{12}$ N. R. Perkins, M. N. Horton, Z. Z. Bandic, T. C. McGill, and T. F. Kuech, Mater. Res. Soc. Symp. Proc. 395, 243 (1996).

${ }^{13}$ W. Shan, T. J. Schmidt, X. H. Yang, S. J. Hwang, J. J. Song, and B. Goldenberg, Appl. Phys. Lett. 66, 985 (1995).

${ }^{14}$ C. C. Klick and J. H. Schulman: Solid State Physics, edited by F. Seitz and D. Turnbull (Academic, New York, 1957), Vol. 5, p. 99.

${ }^{15}$ R. Zhang and T. F. Kuech, J. Electron Mater. (to be published). 